\title{
Progress in Dielectrophoretic Assembly of Carbon Nanotubes for Sensing Application
}

\author{
Hong $\mathrm{ZHANG}^{1, \mathrm{a}}$, Libao $\mathrm{AN}^{2, \mathrm{~b}}$ \\ ${ }^{1}$ College of Life Sciences, North China University of Science and Technology, Tangshan, Hebei \\ 063009, China \\ ${ }^{2}$ College of Mechanical Engineering, North China University of Science and Technology, Tangshan, \\ Hebei 063009, China \\ atzhanghong@ncst.edu.cn, ${ }^{b}$ lan@ncst.edu.cn
}

\begin{abstract}
Carbon nanotubes (CNTs) have shown their potential for broad applications in field effect transistor, field emission, interconnects, energy storage, biomedicine, and many others, due to their excellent electrical, thermal, and mechanical properties. One requirement for many of these applications is to assemble CNTs into designated devices. As a promising nanomanipulation method, dielectrophoresis (DEP) has been widely applied to assemble CNTs in the fabrication of CNT-based nanodevices. This paper presents a brief review of the progress in CNT-based nanosensors and the application of DEP in device making. These sensors focus on the sensing of gases, temperature, bioparticles, light, pressure, stress, and strain.
\end{abstract}

Keyword: carbon nanotube; assembly; dielectrophoresis; application; sensor

\section{Introduction}

Carbon nanotubes (CNTs) have shown excellent properties in electronics, mechanics, and thermotics due to their unique structures. A variety of applications of CNTs has been demonstrated in field effect transistor, field emitter, interconnects, energy storage, and biomedicine [1-3]. Recently, a potential of CNTs as sensors has drawn the attention of many researchers such as for gas sensing, thermal sensing, light sensing, pressure and strain sensing [4-6].

A requirement for many of these applications and potentials is to bridge CNTs across a pair of electric conductors [7]. Several techniques have been used to assemble CNTs onto electrodes, which include using an atomic force microscope (AFM) or scanning tunneling microscope (STM), orientated CNT growth by chemical vapor deposition (CVD), and dielectrophoresis (DEP) under alternating current (AC) electric filed [8]. Among these methods, DEP has been widely applied to manipulate CNTs and other nanorodes, as it does not require special external environment such as high temperature and vacuum and will not destroy CNTs.

In this paper, we introduce DEP theory and its application in manipulating CNTs for sensor fabrication. The DEP parameters which influence the assembly results mainly include the magnitude of the applied voltage, the concentration of the CNT suspension, and 
the duration of the electric field. CNT-based sensors assembled by DEP have displayed their capability in gas sensing, thermal sensing, bio sensing, light sensing, pressure sensing, stress and strain sensing. The paper presents a brief review of the recent development in this field.

\section{DEP Theory}

The DEP takes place when a polarizable particle in a liquid medium is subjected to a non-uniform electric field. In such a circumstance, a dipole moment is induced in the particle and the force acting on each side of the particle will be different, causing the particle to move with respect to the medium. The direction of DEP force depends on the polarizability of the particle relative to that of the medium. If the polarizability of the particle is greater than the polarizability of the medium, the force will push the particle towards the high electric field region and it is called positive DEP. In such a situation, if a pair of electrodes and DEP parameters is properly designed, the particle will move towards the electrode gap and finally deposit across the gap and make a connection of the electrode pair. The DEP force exerted on a polarized particle can be written as [9]

$$
F_{D E P}=(p . \nabla) E,
$$

where $E$ is the strength of the applied electric field, and $p$ is the induced dipole moment.

Before CNTs are assembled by DEP, CNTs are first dispersed in dielectric liquid and separated sufficiently for uniform solution. Then CNT solution is dropped onto the electrode region. Under externally applied AC electric field, CNTs will move towards the electrode gap and finally deposit across the gap, finishing their assembly by DEP.

\section{Gas Sensors}

\subsection{Detection of Ammonia (NH3).}

R. Wang et al used DEP to assemble multi-walled carbon nanotubes (MWNTs) across interdigitated gold electrodes (IDEs) [10]. They demonstrated that the assembled MWNTs could serve as gas sensor for $\mathrm{NH}_{3}$ detection. The electrical conductivity of the MWNT sensor decreased when exposed to $\mathrm{NH} 3$ at room temperature. A good linear correlation between the decreasing amplitude of conductance and the $\mathrm{NH}_{3}$ concentration was observed, and the detection limit of $10 \mathrm{ppm} \mathrm{NH}_{3}$ could be achieved.

$\mathrm{K}$. Xu et al fabricated single-walled carbon nanotube (SWNT)-based $\mathrm{NH}_{3}$ gas sensor using DEP [4]. Their results illustrated that the gas sensor had high sensitivity. The electrical conductance of the sensor reduced two times after exposed to $\mathrm{NH}_{3} \cdot \mathrm{NH}_{3}$ molecules on the surface of SWNTs were removed by ultraviolet (UV) ray irradiation for 10 min. Therefore, their $\mathrm{NH}_{3}$ gas sensor could be reversible. Their experiments showed that amplitude of $10 \mathrm{~V}$ and frequency of $2 \mathrm{MHz}$ of the applied $\mathrm{AC}$ voltage was efficient for SWNT assembly.

\subsection{Detection of Hydrogen ( $\mathrm{H} 2)$.}

Palladium (Pd) has been commonly used for conventional $\mathrm{H}_{2}$ gas sensors and has been incorporated as well with CNT gas sensors for $\mathrm{H}_{2}$ sensing. W. Ding et al. proposed a liquidphase electrochemical reaction method to fabricate a Pd-functionalized $\mathrm{CNT} \mathrm{H}_{2}$ gas sensor 
[11]. SWNTs were assembled onto microelectrodes by DEP. In their experiments, the CNT-retaining electrode was dipped in a Pd acetate solution with a graphite rod as anode. Direct current (DC) voltage was applied between the microelectrode and the graphite rod, and then Pd was deposited onto the surface of SWNTs. Their fabricated CNT-based sensor could respond reversibly to $\mathrm{H}_{2}$ gas in the range of $0.01-1 \%$ concentration, at room temperature in the air.

Graphene oxide (GO) nanostructures have also been fabricated by DEP for $\mathrm{H}_{2}$ gas sensing by J. Wang, et al. [12]. The room temperature experiments showed that their sensor was effective with a sensing response of $5 \%$ and fast response time of less than $90 \mathrm{sec}$. The recovery time was less than $60 \mathrm{sec}$ for $100 \mathrm{ppm} \mathrm{H}_{2}$ gas concentration. The optimum DEP parameters for manipulating GO nanostructures were peak-to-peak voltage of $10 \mathrm{~V}$ and frequency of $500 \mathrm{kHz}$, when manipulation time was $30 \mathrm{sec}$.

\subsection{Detection of other Gases.}

M. Fujioka et al. made use of DEP to separate semiconducting SWNTs from commercial CNT mixtures and then further enriched them by using a spin column and dextran-based gel for SWNT-based sensor fabrication [13]. The sensor fabricated using separated semiconducting SWNTs was more sensitive against nitrogen dioxide $\left(\mathrm{NO}_{2}\right)$ gas by two times than that fabricated using the pristine SWNTs mixture.

Y. Dan et al. used DEP to assemble polymer nanowires onto gold electrodes for gas sensing [14]. Their sensor showed a resistance change of $10.5 \%, 9 \%$, and $4 \%$ at the saturation vapor pressure of acetone, methanol and ethanol, respectively. The response and recovery time of the sensor were in second level with excellent reproducibility.

\section{Thermal Sensors}

V. Agarwal et al. incorporated SWNTs onto CMOS circuitry for fully functional CNTbased thermal sensor fabrication by DEP [5]. They pretreated the CMOS chip to prepare the top metal layer for DEP using an electroless zincation. After CNT deposition, The sensor was encapsulated with a parylene-C layer to reduce the contact resistance of CNTs with electrodes. Experiments indicated that the temperature coefficient of resistance for the sensor was $-0.40 \%$ from $25^{\circ} \mathrm{C}$ to $105^{\circ} \mathrm{C}$. Their concept could be extended for other applications.

S. Selvarasah et al. proposed a hybrid fabrication technique for 3-D thermal sensors based on CNTs [15-16]. First, a 2 mask process was utilized to make a microplatform. Then CNTs were assembled onto the platform by DEP. Encapsulation with a thin parylene layer reduced the contact resistance and improved the performance of the sensor. The temperature coefficient of resistance of their MWNT-based thermal sensor was measured to be between $-0.21 \%$ and $-0.66 \%$ per degree. [15]. For their SWNT-based thermal sensor, the temperature coefficient of resistance ranged from $-0.154 \%$ to $-0.24 \%$ for the single electrode device and varied from -0.3 to $-0.57 \%$ for the multielectrode device [16].

\section{Bio Sensors}

J. Clendenin fabricated a biosensor based on SWNTs with integrated single-strand DNAs (ssDNA) using DEP [17]. The sensor provided a label-free electronic detection of DNA hybridization between surface-immobilized ssDNA and target ssDNA in real time. Hybridization kinetics between complementary and target ssDNA nucleotide base pairs 
generated local charges, which brought a measurable electrical conductance change in SWNTs.

J. Suehiro et al. proposed a method for bacteria detection using a CNT-based gas sensor, which was fabricated by DEP assembly and incorporated with a microheater in a small chamber into a bio-microelectromechanical system (Bio-MEMS) [18]. Bacteria were heated by the heater to generate $\mathrm{NH}_{3}$ gas by oxidation reaction, and then $\mathrm{NH}_{3}$ gas was detected by the sensor. It was found that the CNT-based sensor could detect and quantify $10^{7}$ bacteria cells.

\section{Light Sensors}

CNTs have a potential as infrared (IR) detection materials due to their distinctive electronic properties. J. Zhang et al. utilized a DEP system and an Atomic Force Microscopy (AFM) based nanomanipulation system for controllable and reliable position of an individual MWNT onto the electrodes for IR detection [19]. The diameter of a single MWNT was modified by electrical breakdown of its outer walls to tune its electronic property for better performance. The single MWNT-based infrared sensor showed higher quantum efficiency than the SWNT-based sensors.

J. Lee et al. fabricated GaN Nanowire (NW) devices with back-gated structure using DEP for ultraviolet sensing [6]. A considerable increase of conductance was detectd under UV light with wavelengths of 365 and $254 \mathrm{~nm}$.

\section{Pressure/Stress/Strain Sensors}

\subsection{Pressure Sensors}

By using DEP and a MEMS-compatible process, C.K.M. Fung et al. incorporated CNT strands as sensing elements onto arrays of Polymethylmethacrylate (PMMA) diaphragms for pressure sensing [20]. The piezoresistive effects of the CNT sensing elements were measured and results showed that the sensor was able to detect the variation of the input pressure. Moreover, the piezoresistive gauge factor of the CNT-based sensor might be much greater than polysilicon based sensors.

\subsection{Stress Sensors}

W.W.Y. Chow et al. used DEP to manipulate SWNT bundles on PMMA substrate in an array for gas-flow shear stress sensing [21]. The sensor detected gas-flow in a PMMA microchannel by means of thermal transfer principle. Under constant temperature, the voltage output of the sensor increased with the increasing flow rate in the microchannel. Experiments revealed that the power of the sensor had a linear relation with one third of the power of the shear stress, and the sensor was more sensitive to the flow with a higher overheat ratio.

\subsection{Strain Sensors}

J. Tong et al. utilized DEP for batch microfabrication of strain sensors using silicon cantilevers with MWNTs as sensing elements [22]. Based on their investigation, the CNTbased cantilevers displayed a linear relationship between externally applied strain and electric resistance change. For four tested electrode configurations, the gauge factor was from 78.84 to 134.40 in their experiments. 


\section{Conclusions}

Recent studies show that CNTs can be used as gas sensors for $\mathrm{NH}_{3}, \mathrm{H}_{2}, \mathrm{NO}_{2}$, acetone, methanol, and ethanol gas detection, as thermal sensors for temperature detection, as biosensors for DNA and bacteria, have the ability to distinguish infrared and ultraviolet lights, and can be serve as sensors for pressure, stress, and strain sensing. The unique nanostructure and properties of CNTs provide great opportunities towards extreme miniaturization, high sensitivity, rapid response, and low power consumption of the CNTbased sensors. DEP technique offers a quick and pollution-free batch manipulation of CNTs in sensor-making process, accelerating the industrial applications of CNTs in sensing and many other fields.

\section{Acknowledgments}

The research was sponsored by the National Natural Science Foundation of China (51172062, 51472074), and the Hundred Talents Program of Hebei Province of China (E2012100005).

\section{References}

1. P. Stokes and S.I. Khondaker, High quality solution processed carbon nanotube transistors assembled by dielectrophoresis, Appl. Phys. Lett. 96 (2010) 083110-1-3.

2. G. Chai, L. Chow, D. Zhou, S.R. Byahut, Focused-ion-beam assisted fabrication of individual multiwall carbon nanotube field emitter, Carbon 43 (2005) 2083-2087.

3. P. Makaram, S. Selvarasah, X. Xiong, et al., Three-dimensional assembly of singlewalled carbon nanotube interconnects using dielectrophoresis, Nanotechnology 18 (2007) 395204-1-5.

4. K. Xu, X. Tian, C. Wu, et al., Fabrication of single-walled carbon nanotube-based highly sensitive gas sensors, Sci. China Tech. Sci. 56 (2013) 32-35.

5. V. Agarwal, C.L. Chen, M.R. Dokmeci, S. Sonkusale, A CMOS integrated thermal sensor based on single-walled carbon nanotubes, IEEE Sensors Conference (2008) 748-751.

6. J. Lee, K. Moon, M. Ham, J. Myoung, Dielectrophoretic assembly of GaN nanowires for UV sensor applications, Solid State Commun. 148 (2008) 194-198.

7. L. An and C.R. Friedrich, Process parameters and their relations for the dielectrophoretic assembly of carbon nanotubes, J. Appl. Phys. 105 (2009) 074314-17.

8. L. Feng, L. An, C.G. Lu, C.W. Lu, Progress towards the automatic assembly of carbon nanotubes by dielectrophoresis, Advanced Materials Research 418-420 (2012) 731-734.

9. J.E. Kim and C.S. Han, Use of dielectrophoresis in the fabrication of an atomic force microscope tip with a carbon nanotube: a numerical analysis, Nanotechnology 16 (2005) 2245-2250.

10. R. Wang, H. Li, M. Pan, D. Chen, A carbon nanotube gas sensor fabricated by dielectrophoresis and its application for NH3 detection, Proceedings of the 31st Annual International Conference of the IEEE EMBS., Minneapolis, USA (2009) 6046-6049.

11. W. Ding, R. Hayashi, J. Suehiro, et al., Calibration methods of carbon nanotube gas 
sensor for partial discharge detection in $\mathrm{SF}_{6}$, IEEE T. Dielect. El. In. 13 (2006) 353361.

12. J. Wang, B. Singh, J. Park, et al., Dielectrophoresis of graphene oxide nanostructures for hydrogen gas sensor at room temperature Sensor. Actuat. B-Chem. 194 (2014) 296-302.

13. M. Fujioka, H. Watanabe, Y. Martin, M. Nakano, Separation and enrichment of semiconducting carbon nanotubes and its application to highly sensitive carbon nanotube gas sensor, Proceedings of the 2011 IEEE Nanotechnology Materials and Devices Conference, Jeju, Korea (2011) 403-407.

14. Y. Dan, Y. Cao, T.E. Mallouk, A.T. Johnson, S. Evoy, Dielectrophoretically assembled polymer nanowires for gas sensing, Sensor. Actuat. B-Chem. 125 (2007) 55-59.

15. S. Selvarasah, P. Makaram, C.L. Chen, et al., A three dimensional multi-walled carbon nanotube based thermal sensor on a flexible parylene substrate, Proceedings of the 7th IEEE International Conference on Nanotechnology, Hong Kong, China (2007) 1062-1066.

16. S. Selvarasah, C.L. Chen, S.H. Chao, et al., A three dimensional thermal sensor based on single-walled carbon nanotubes, Proceedings of the 14th International Conference on Solid-State Sensors, Actuators and Microsystems, Lyon, France (2007) 1023-1026.

17. J. Clendenin, J. Kim, S. Tung, An aligned carbon nanotube biosensor for DNA detection, Proceedings of the 2nd IEEE International Conference on Nano/Micro Engineered and Molecular Systems, Bangkok, Thailand (2007) 1028-1033.

18. J. Suehiro, N. Ikeda, A. Ohtsubo, K. Imasaka, Bacterial detection using a carbon nanotube gas sensor coupled with a microheater for ammonia synthesis by aerobic oxidization of organic components, IET Nanobiotechnol. 3 (2009) 15-22.

19. J. Zhang, N. Xi, H. Chen, K.W.C. Lai, Fabrication and experimental testing of individual multi-walled carbon nanotube (CNT) based infrared sensors, Proceedings of the 6th Annual IEEE Conference on Sensors, Atianta, USA (2007) 511-514.

20. C.K.M. Fung, M.Q.H. Zhang, Z. Dong, W.J. Li, Fabrication of CNT-based MEMS piezoresistive pressure sensors using DEP nanoassembly, Proceedings of the 5th IEEE Conference on Nanotechnology, Nagoya, Japan, 1 (2005) 199-202.

21. W.W.Y. Chow, W.J. Li, S.C.H. Tung, Integrated CNT sensors in polymer microchannel for gas-flow shear-stress measurement, Proceedings of the 3rd IEEE Int. Conf. on Nano/Micro Engineered and Molecular Systems, Sanya, China (2008) 10111014.

22. J. Tong, M. Priebe, Y. Sun, Carbon nanotube-based strain sensing cantilevers, IEEE 20th International Conference on Micro Electro Mechanical Systems (MEMS 2007), Kobe, Japan (2007) 843-836. 